\title{
Electronic states on a fractal: The consequences of self-energy variation
}

\author{
R. F. S. Andrade \\ Instituto de Física, Universidade Federal da Bahia, 40210-340, Salvador, Brazil \\ H. J. Schellnhuber \\ Potsdam Institute for Climate Impact Research, 14412, Potsdam, Germany
}

(Received 26 August 1996)

\begin{abstract}
A tight-binding model on the Koch curve with different self-energies on certain sites is investigated. The energy spectrum and the wave functions are analyzed by means of transfer matrices. An exact analysis of the band splitting process leads to a precise classification of the band-edges states, which fall into three infinite subsets formed by extended, localized, and fast growing self-affine states. Numerical investigation indicates that the model also admits an infinite number of exotic states. The present results are compared with those of a previously analyzed tight-binding model with next-nearest-neighbor interactions on the same fractal. A comparative discussion of these results distinguishes spectrum and state properties dependent on the details of the model from those which are common to both models and depend only on the geometry of the fractal. [S0163-1829(97)01519-1]
\end{abstract}

\section{INTRODUCTION}

The investigation of electronic states on nonramified fractals $^{1,2}$ profits from the huge amount of new methods which have been developed and information which has been gathered for quantum systems in aperiodic linear chains. ${ }^{3-6}$ The fractal dimension which characterizes the geometry does not play a relevant role for the dynamics of models constructed on such sets, which can be shown to be topologically equivalent to one-dimensional structures. On the other hand, the scale invariance associated with the geometry of the fractal can be used to construct models where a nonperiodic ordering is superimposed upon one-dimensional structures, such as the aperiodic linear chains which have proven very useful in mimicking the effect of disorder in physical systems. $^{7}$

The results obtained for aperiodic chains have identified their characteristic types of quantum states (extended, critical, or localized) and the correponding nature of the energy spectrum (absolutely continuous, singular continuous, or pure point). The occurrence of different types of states in different models depends on some global features of the aperiodic potential of the model, in particular, whether it is continuous $^{3}$ or has finite ${ }^{5}$ or infinite ${ }^{8}$ discontinuities. It is also found that the existence of a finite threshold value of the potential for a transition in the character of the states depends on these general features of the potential. Despite huge progress made recently in the classification of states, however, the picture is not yet complete, and it is expected that the investigation of further aperiodic or quasiperiodic models (e.g., those obtained by hyperinflation rules ${ }^{9}$ ) will reveal additional qualitative and quantitative phenomena.

Undoubtedly, the most important innovation in the methods used to analyze quasiperiodic chains is the introduction of concepts of dynamical systems analysis. ${ }^{5}$ These methods, which were developed to study the trajectories of nonlinear maps in phase space, often provide rigorous results in the quantum case too. The approach takes advantage of the for- mulation of the Schrödinger equation of discrete tightbinding models in terms of transfer matrices (TM's) and derives maps which relate elements of the TM's of successive generations of the construction of the aperiodic model in terms of periodic approximations. This scheme has an even broader applicability since the continuous Schrödinger equation can be exactly transformed into discrete tight-binding form. ${ }^{10}$

Among the main results which were obtained by using these tools we mention the finding that the Cantor set structure of the energy spectrum ${ }^{11,12}$ can often be induced from the iteration of a low-dimensional system of discrete maps (in the case of models based on the Fibonacci sequence this system is actually reduced to a single map $^{5}$ ). The invariant sets of maps involved, like fixed points, higher-order periodic cycles, or higher-dimensional invariant manifolds, are related to certain quantum states and their several pertinent properties. Particularly it has been shown that higher-order cycles are related to the energy dependent scaling exponent of the spectrum, e.g., for the Fibonacci sequence based models, cycles of order six and two controls, respectively, scaling at center and edge of the bands. ${ }^{13-15}$ Scaling behavior of critical wave functions also follow from the eigenvalues of the linear stability analysis of the invariant sets. ${ }^{13}$ The localization length of the states in question is related to the Lyapunov exponent evaluated along the orbits of these maps, ${ }^{15}$ while multifractal measures can be used to identify their character. ${ }^{16}$

A second important method is provided by renormalization transformations for the evaluation of the Green's functions associated with the Hamiltonian under investigation. The successive decimation of sites in the chain, generating a renormalization of the site energies and hopping terms, leads to a virtual decoupling of the sites from their neighbors. In the infinite limit of decimation the contribution of all sites of the chain to the Green's function is collapsed onto the surviving diagonal element. This method had been first introduced for analyzing the effect of configurational disorder in 
random systems, ${ }^{17}$ but has been latter applied with success to the analysis of fractal structures, ${ }^{18,19}$ hierarchical lattices, ${ }^{20-22}$ or deterministically disordered chains ${ }^{23,24}$ where exact renormalization schemes may be established.

A tight-binding model based on the aperiodicity defined by a certain nonramified fractal (the Koch curve) has been analyzed previously, in an exemplary way, by the authors with the help of both transfer matrix ${ }^{25,26}$ and Green's function $^{27}$ methods. This model includes nearest-neighbor hopping for every site as well as next-nearest-neighbor (NNN) hopping for a well-defined set of sites, which depend on the law of generation of this geometrical fractal. The results indicate that the system supports an extraordinarily rich variety of states, whose main properties can be either obtained analytically or may be computed numerically with high accuracy. Among the most important aspects we may quote the abundance of critical and localized states; ${ }^{25}$ the absence of a finite threshold value of the NNN hopping strength for the change in the character of quantum states ${ }^{25,26}$ a precise classification scheme for the quantum states of the model, based on the four-adic expression of the values of the integrated density of states $\kappa ;{ }^{26}$ and the exact evaluation of the density of states, taking into account all the different contributions of nonequivalent sites for the Green's function. ${ }^{27}$

This series of investigations laid out in detail several properties of a single model, but left open the important question of whether the properties of the quantum states are generic for that particular geometry or whether some of them are dependent on the details of the model. A definite answer to this question, if available, is almost as important as the solution of a large set of models. A most convenient way to side step the typical difficulties of finding exact results to generic problems is to gather evidence through the actual analysis of several model cases. In this work we follow this strategy. To resume the discussion we consider the most simple but similar tight-binding model on the same nonramified Koch fractal, where the perturbations which distinguish it from the linear chain are caused by different values of self-energies. ${ }^{24}$ This form limits the effect of nonperiodicity to single sites, and is able to give valuable answers to such typical questions stated above.

This same question has been considered for models defined on the basis of the Fibonacci sequence. For the model first considered ${ }^{5}$ that sequence defines different on-diagonal self-energies, whereas for a second model ${ }^{13,28}$ it prescribes different strengths for hopping to neighboring sites. The common geometric aspect to both models leads to several identical properties (e.g., the form of the trace map), but the different initial conditions for mapping reflect different properties of individual quantum states.

Our model is analyzed with the help of some of the methods used in our prior investigations: the formulation of the Schrödinger equation in terms of TM's within the scheme of periodic approximation, the exploration of invariant sets of the nonlinear maps which link the matrix elements of the TM's of successive generations, the identification of the character of the quantum states by the evaluation of the band-gap ratio, and also by the numerical integration of the corresponding Schrödinger equation. This work gives some definite answers to the main question stated above (e.g., a classification of the character of the states based on the fouradic expression of the value of $\kappa$ ), while the character of the states, at least for a large subset, does depend on the details of the model.

The rest of this paper is organized as follows: in Sec. II we introduce the model Hamiltonian and obtain the pertinent maps for the matrix elements of the TM's within the scheme of periodic approximations. In Sec. III we discuss the spectrum, the properties of the invariant sets, and the way they are reflected in the subset of extended states. We also present the results of numerical investigation which indicate the existence of subsets of localized and critical states. Finally, Sec. IV closes the paper with a comparison of the present results with those obtained for the NNN system.

\section{MODEL}

As anticipated in the preceding section, we define the model with the same basic elements used in the NNN system. We introduce the one-parameter Hamiltonian

$$
\mathbf{H}_{N}=\sum_{n \in Z} \lambda t_{N}(n)|n\rangle\langle n|+\sum_{n \in Z}[|n\rangle\langle n-1|+| n\rangle\langle n+1|],
$$

where we assume that the system is being constructed by periodic approximations labeled by $N$. The unit cell in each approximation corresponds to the figure obtained in the $N$ th generation of the construction of the fractal. ${ }^{25} t_{N}(n)$ is the same function which selects those sites at the acute angles of the Koch curve used in the NNN system and is expressed by

$$
\begin{gathered}
t_{N}(n)=\delta(n, 0)+\sum_{s=1}^{N-1} \delta\left(4^{s} / 2, n\left[\bmod 4^{s}\right]\right), \\
-\frac{1}{2} 4^{N}<n \leqslant \frac{1}{2} 4^{N}, \quad t_{N}\left(n+4^{N}\right)=t_{N}(n) \quad \forall n \in Z .
\end{gathered}
$$

Deterministic disorder may appear in Eq. (2.1) as ondiagonal autoenergy terms of magnitude $\lambda$. The general form of the Schrödinger equation following from Eq. (2.1) is

$$
a_{m-1}+\left[\lambda t_{N}(m)-E\right] a_{m}+a_{m+1}=0 .
$$

We make use of the formalism of transfer matrices and write Eq. (2.3) as

$$
u_{m+1}=T_{m} u_{m},
$$

where

$$
u_{m}=\left(\begin{array}{c}
a_{m} \\
a_{m-1}
\end{array}\right), \quad T_{m}=\left(\begin{array}{cc}
\tau_{m} & -1 \\
1 & 0
\end{array}\right),
$$

with

$$
\tau_{m}=E \quad \text { if } t_{N}(m)=0, \quad \tau_{m}=E-\lambda \quad \text { if } t_{N}(m)=1 .
$$

We observe that the matrices $T_{m}$ have only two different forms, henceforth called $R$ and $Q$, according to whether $t_{N}(m)$ equals 0 or 1 . With the help of Eq. (2.4) it is possible to obtain a single TM, expressed by the product of $p$ matri- 
ces $T_{m}$, which relates the vectors $u_{m+p}$ with $u_{m}$ for any value of $p$. In particular, for each periodic approximation $N$, we can obtain matrices $A_{N}$ which describe the effect of a whole unit cell in that approximation. It is straightforward to observe that the $A_{N}$ obey the following recurrence rule, which is characteristic for this fractal ${ }^{25,29}$

$$
A_{N+1}=A_{N}^{2} B A_{N}^{2},
$$

where $A_{0}=R$ and $B=R^{-1} Q$.

All relevant information concerning the band structure and quantum states of the model is contained in the maps for the matrix elements which are derived from Eq. (2.7). Due to the conservation of probability flow, which requires $\operatorname{det} A_{N}=1$, it is possible to derive a closed system of only two mapping equations in terms of the variables $\xi_{N}=\operatorname{Tr} A_{N}$ and $\varphi_{N}=\left(A_{N}\right)_{12}$. They read

$$
\begin{aligned}
\xi_{N+1}= & \left(\xi_{N}^{2}-2\right)^{2}-2+\lambda \varphi_{N} \xi_{N}\left(\xi_{N}^{2}-2\right), \\
& \varphi_{N+1}=\varphi_{N} \xi_{N}\left(\xi_{N}^{2}-2\right)+\lambda \varphi_{N}^{2} \xi_{N}^{2} .
\end{aligned}
$$

The map (2.8) has some similarities with its counterpart in the case of the NNN system. However, as we will see in the next section, the invariant sets of the map for the old and new models have quite different properties and it is natural that this will be reflected in the nature of the quantum states. Nevertheless it turns out that some of the properties of the NNN system which were related to its attracting sets still persist in the present case, in spite of the fact that the dynamics of the new maps are different.

\section{SPECTRUM, INVARIANT SETS, AND QUANTUM STATES}

\section{A. Spectrum}

The band splitting process in successive periodic approximations $N$ is dictated only by the behavior of Eq. (2.8). For each $N$, the spectrum $\sigma_{N}$ is the set of energy values for which $\left|\xi_{N}(E)\right| \leqslant 2$. It is obtained after the successive iterations of Eq. (2.8), subject to the initial conditions

$$
\xi_{0}=E, \quad \varphi_{0}=-1 .
$$

It is straightforward to certify that, for any value $\lambda \neq 0$, the system (2.8) leads to a band splitting process similar to that obtained for the NNN system: each subband of the $N$ th generation will split into four subbands in the $N+1$ th generation, each one containing $1 / 4$ of the states of the $N$ th generation subband. Thus, in the limit $N \rightarrow \infty, \sigma_{N}$ has exactly the same kind of Cantor-set-like structure ${ }^{30}$ which was found for the NNN system: it is quite clear that this property is directly linked to the geometry of the fractal substrate, or more specifically, to the rule used to introduce aperiodic perturbations on the periodic structure.

\section{B. Invariant sets}

Information about the nature of the quantum states follows from the direct integration of the Schrödinger equation or from the analysis of the invariant sets of Eq. (2.8). When $\lambda=0$ the system (2.8) has three fixed points (FP's), namely $(2,0),[(-1 \pm \sqrt{5}) / 2,0]$, and a fixed line $(-1, \varphi)$. The inter- section of the fixed line with the plane $\varphi=0,(-1,0)$, and the three FPs are related to a trivial map of the homogeneous chain onto itself, in which we perform a decimation of three out of each four original sites. When $\lambda \neq 0$ the only change in the form of the invariant sets is the restriction of the line $(-1, \varphi)$ to the FP $(-1,0)$. This indicates that all dynamical properties of the invariant sets of Eq. (2.8) reflect the properties of the quantum states of the linear chain, thus they are related to the occurrence of extended states. This fact contrasts with what was observed in the analysis of the NNN system: the presence of exotic or critical quantum states was anticipated by the presence of new invariant sets which vanished when the fractal model collapsed into the ordinary linear chain system for $\lambda \rightarrow 0$.

The eigenvalue spectra of the linear stability analysis of the four FP's show that all of them are unstable, having at most one neutral and one unstable eigenvector, so that no orbit can be progressively attracted by any of them. The other possibility of starting exactly on the FP's, must also be discarded as it is not compatible with the initial conditions (3.1). Therefore, these FPs can only be linked to extended states whose corresponding orbits, described by Eq. (2.8), jump from far away in the phase space directly into them. The possible existence of such orbits can be better investigated with the help of the backward iteration maps, which are obtained by inverting (2.8) as

$$
\begin{gathered}
\xi_{N}= \pm \sqrt{2 \pm \sqrt{Z_{N+1}}}, \\
\varphi_{N}=\frac{\xi_{N+1}+2-Z_{N+1}}{\lambda \xi_{N}\left(\xi_{N}^{2}-2\right)},
\end{gathered}
$$

where

$$
Z_{N+1}=\frac{\left(\xi_{N+1}+2\right)^{2}}{\xi_{N+1}+2+\lambda \varphi_{N+1}} .
$$

Let us suppose that the $N+1$ th iteration of a given orbit will reach one of the FP's, that is, $\left(\xi_{N+1}, \varphi_{N+1}\right)=(\bar{\xi}, 0)$, where $\bar{\xi}$ is any of the four possible values listed above. Then (3.2)-(3.4) indicate that $\varphi_{N}=0$, and $\xi_{N}$ may assume four different values, one of which is $\xi_{N+1}$ itself. Further preimages of these preimages can be evaluated with the help of (3.2)-(3.4), and we soon recognize that all points of this set are of the form $(\xi, \varphi)=(\xi, 0)$. Since the points visited by a true orbit generated with Eq. (2.8) have $\varphi \neq 0$, the set of successive preimages does not bring much relevant physical information.

An important exception to this general situation emerges from a closer analysis of $\left(\xi_{N+1}, \varphi_{N+1}\right)=(2,0)$. In this case the solutions of Eqs. (3.2) and (3.3) are $\left(\xi_{N}, \varphi_{N}\right)=(2,0) ;(-2,0)$ and $\left(0, \varphi_{N}\right)$ (double degenerated). For this last situation the whole line $\left(\xi_{N}, \varphi_{N}\right)=\left(0, \varphi_{N}\right)$ will be mapped onto $(2,0)$ in the next iteration. For a fixed value of $\lambda$ this line certainly includes all subband centers, which become fixed band-end points for all further generations. A detailed discussion of the effect of this dynamics in the classification of the quantum states is given below. Besides this, it is possible to identify a further set of points which will be mapped onto $(2,0)$ : it is the set of all preimages of the point $\left(\xi_{N}, \varphi_{N}\right)=(-2,0)$. In the evaluation of the preimages of this 
point we observe that the equations (2.8) become degenerated, so that the inverse-iteration expressions (3.2-3.4) are no longer correct. The evaluation of the preimages of $(-2,0)$ goes along a path of its own, indicating that a whole line of points, expressed by the relation

$$
\lambda \varphi_{N-1} \xi_{N-1}+\xi_{N-1}^{2}-2=0,
$$

will be mapped onto $(2,0)$ after two iterations.

\section{Quantum states}

As mentioned before, the identification of the nature of quantum states follows from the investigation of the Schrödinger equation and of the properties of the invariant sets. The relevant quantum number for the identification of the states of quasiperiodic operators is the quantum rotation number $\kappa(E)$ defined as ${ }^{30,31}$

$$
\kappa(E)=\frac{1}{\pi} \int_{-\infty}^{E} \rho(\bar{E}) d \bar{E}
$$

$\kappa(E)$ is a well defined function of $E$ in each finite order periodic approximation and also in the limit $N \rightarrow \infty$, when the density of states $\rho(E)$ becomes a sum of $\delta$ spikes. In order to simplify the discussion of the results let us assume $\lambda>0$. This represents no restriction, since we observe from Eq. (2.8) that $\xi_{N+1}(E, \lambda)=\xi_{N+1}(-E,-\lambda)$, so that if $(E, \lambda)$ belongs to the spectrum the same is true for $(-E,-\lambda)$. Also the quantum states associated with $(E, \lambda)$ and $(-E,-\lambda)$ have the same properties. The relation between the values of $\kappa$ for these two states is

$$
\kappa(E, \lambda)=1-\kappa(-E,-\lambda)
$$

Understanding the link between the invariant sets and a few quantum states in the first iterate offers a general scheme for the classification of a large set of extended quantum states. Indeed, the effect of the first iteration of Eq. (2.8) on the system, which can be described analytically, is typical of the higher-order iterations. An analogous treatment becomes impossible in the further successive iterates, where the degree of the pertinent expressions increases exponentially with $N$.

In the first iteration the energy band of the linear chain $[-2,2]$ is split into four subbands. If we make use of the general discussion of the preimages of the FP's we observe that the band center $E=\xi_{0}=0$ will be mapped onto $(2,0)$, where it becomes a band edge in all future iterations. The expressions of the other three roots of $\xi_{1}=2$ are not simple and, since they do not satisfy $\varphi_{1}=0$, they do not correspond to FP's. The same is true for the side centers $E= \pm \sqrt{2}$, which will be mapped onto $(-2,2 \lambda)$. On the other hand, we note immediately that the other two roots of $\xi_{1}=-2$ are

$$
\xi_{0}=\frac{\lambda \pm \sqrt{8+\lambda^{2}}}{2}
$$

These are precisely the roots of Eq. (3.5) with $N=0$, i.e., $\varphi_{0}=-1$, and therefore will become fixed band edges at $(2,0)$ in the second iteration. Moreover it is straightforward to realize that gaps are opened to the right of the centers and side centers of the band $[-2,2]$ and to the to the left of the points given by Eq. (3.8). So we conclude that the right edge of the second and the left edge of the second and fourth subbands will stay on the FP $(2,0)$ for all further iterations $N \geqslant 2$. The values of $\kappa$ corresponding to the three states attracted by $(2,0)$ are, respectively, $\kappa=0.5_{-}, 0.25_{+}$, and $0.75_{+}$. Since the value of $\kappa$ remains constant within the gaps, the indices + or - indicate whether the value of $E$ corresponding to that $\kappa$ is approached from the right-hand or left-hand side. In this notation, the values of $\kappa$ corresponding to the side centers of the zeroth-order generation are $0.25_{-}$ and $0.75_{-}$. These band edges do not remain at a fixed value of energy in successive iterations, and their behavior is not dictated by the properties of any FP.

This same situation will be repeated over and over again in the successive iterations of the map (2.8): when any subband is split into four it gives rise to three new values of the energy which are attracted to the FP $(2,0)$. The corresponding values of $\kappa$ can be obtained by adding to the value of $\kappa$ at the edge of the subband in the $N$ th generation the relative contribution of the $N+1$ th. This is given by the same scheme as before, but dividing those values by $4^{N}$. So we conclude that in the $N$ th generation the values of $\kappa$ for these states are

$$
\begin{gathered}
\kappa=\left(\frac{1}{2} \frac{2 n-1}{4^{N-1}}\right)_{-}, \quad n=1,4^{N-1} \\
\kappa=\left(\frac{2 n-1}{4^{N}}\right)_{+}, \quad n=1,2 \times 4^{N-1},
\end{gathered}
$$

where the indices + and - have the same meaning as above. As we have mentioned, the nature of these states is dictated by the properties of the FP $(2,0)$, which stems from the linear chain. Therefore all of them should be extended. This is indeed the case, as can be confirmed by the results obtained from the band-gap ratio ${ }^{32}$ and the direct integration of the Schrödinger equation (2.3). We also observe that the fouradic expression of all values of $\kappa$ in the above sets has a finite tail.

In addition to this set of extended states, the system admits a set of exponentially localized states. As expected, these states are not related to the FP's, and have been found after several numerical investigations. They correspond to the generalization of those band side-center states that, in the first iteration, are mapped onto $(-2,2 \lambda)$. Thus, they are placed just across some of the main energy gaps, each having an extended state as its immediate neighbor on the other gap side. The values of $\kappa$ for these states are related to those in Eq. (3.10) and are given by

$$
\kappa=\left(\frac{2 n-1}{4^{N}}\right)_{-}, \quad n=1,2 \times 4^{N-1} .
$$

The localized character of the state has been predicted by the band-gap ratio, which vanishes exponentially, and is confirmed by the integration of Eq. (2.3), as illustrated by Fig. 1.

We now turn to analyze the character of the states associated with the subset 


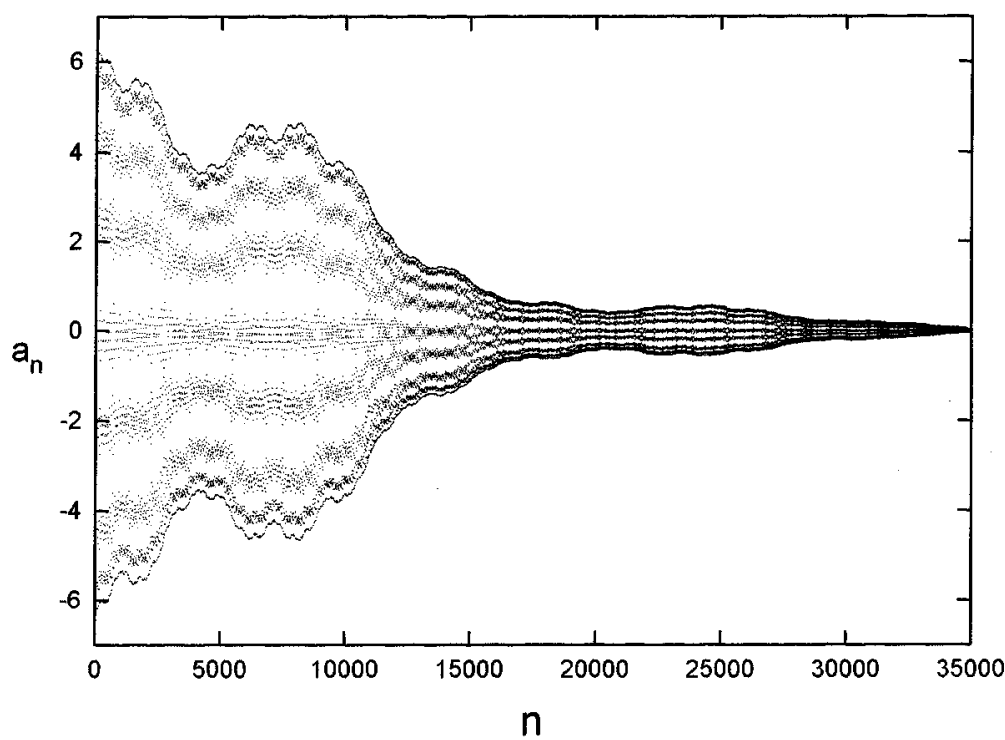

FIG. 1. Localized even wave function for

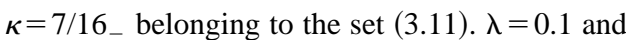
$E=0.4203437660 \ldots$.

$$
\kappa=\left(\frac{1}{2} \frac{2 n-1}{4^{N-1}}\right)_{+}, \quad n=1,4^{N-1}
$$

Their elements share the same property as those of the set (3.11): they are band-edge states which each have an extended state as its immediate neighbor on the other gap side. So it could be argued in a heuristic way that the states of this subset have the same properties as those of Eq. (3.11), much as the states of the sets (3.9) and (3.10) have similar properties. The numerical evaluation of the band-gap ratio supports this argument in the sense that it decreases quite rapidly when $N$ increases. However the integration of Eq. (2.3) uncovers a completely different behavior for the corresponding state, whose typical traits are illustrated by Fig. 2 . The most striking feature is its divergent amplitude, present in both even and odd states, which increases faster than any power law. However, the log-log plot of the wave function shows that the amplitude does not grow in an exponential way, but suggests that it has an envelope growing like $\ln \left(a_{n}\right) \sim(\ln n)^{p}$. This is a hint that the behavior of the band-gap ratio should not decrease in an exponential way, but should, however, decrease faster than any power of $N$. The relatively small number of approximations $(\sim 10)$ where it has been possible to evaluate that ratio do not allow its analytic dependence on $N$ to be obtained in an accurate way. Figure 2 also shows that these states are not self-similar but they are self-affine. The regularity with which the structures of both the envelope and the inner part are reproduced is quite impressive. To our knowledge, quantum states with similar properties have not been reported in any discussion of dynamical properties of quasiperiodic quantum Hamiltonians.

Our further numerical investigation has identified states with similar features to those of the set (3.12) for several other values of $\kappa, \quad$ e.g., $\kappa=2 / 3, \quad \lambda=0.73$, $E=1.2225360933648 \ldots$. We observe that the four-adic expression of $2 / 3$ is $0.22222 \ldots$. Also other states identi-

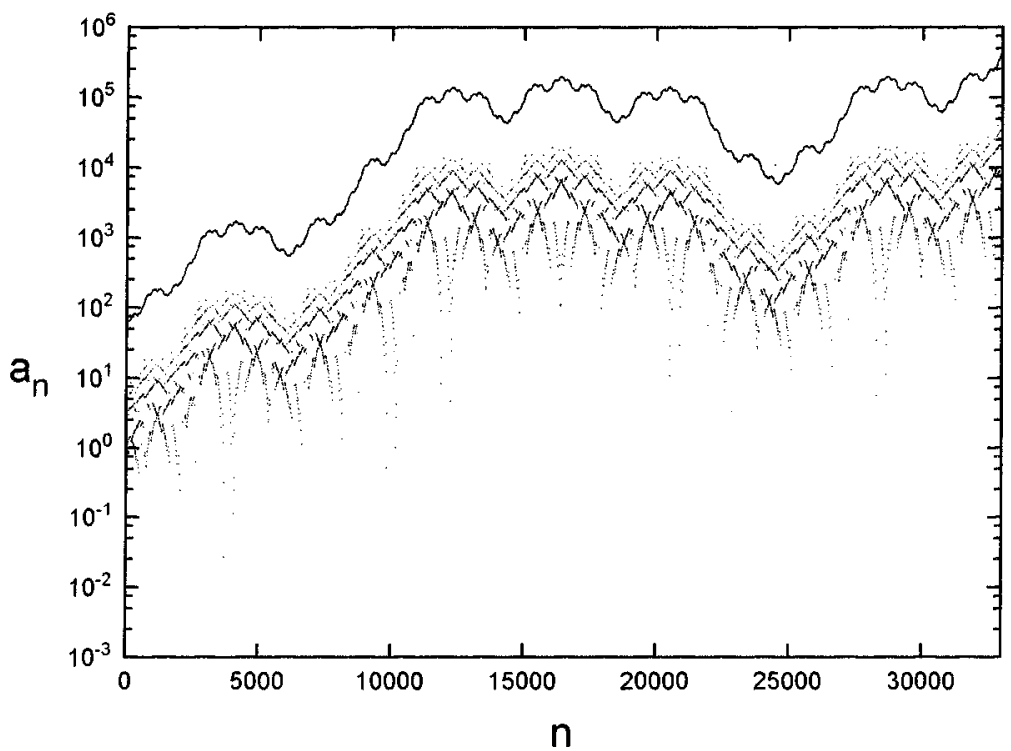

FIG. 2. Fast increasing even wave function for $\kappa=1 / 2_{+}$belonging to the set (3.12). $\lambda=0.1$ and $E=0.06644514714 \ldots$. It has a self-affine profile with two different scaling factors, valid both for the envelope and smaller spikes. 


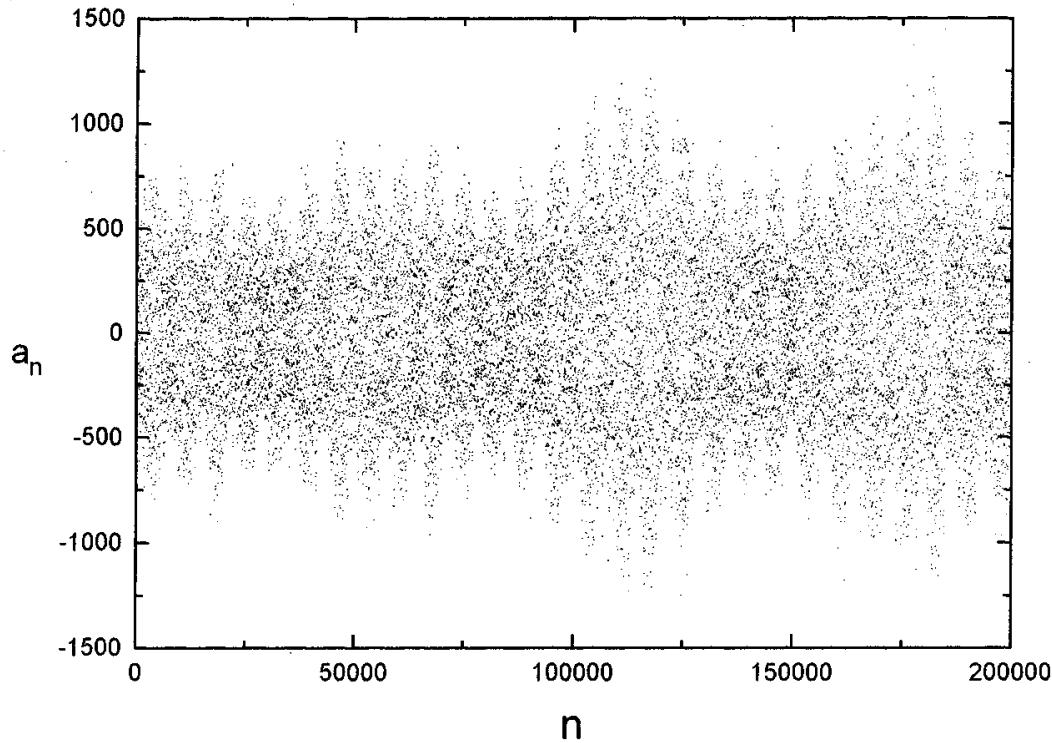

FIG. 3. Exotic even wave function for $\kappa=4 / 7$, which has the four-adic periodic tail $\{210\}$. The band-gap ratio, evaluated with $N \leqslant 25$, seems to converge very slowly to a fixed value. $\lambda=0.73$ and $E=0.738969605897 \ldots$.

fied by values of $\kappa$ with the same tail share this same behavior.

Finally, let us discuss the occurrence of exotic or critical states. First of all it is important to recall that such states on the NNN system are associated with the existence of a critical FP, which is absent in the present case. So the identification of such states proceeds only through the evaluation of the band/gap ratio and the integration of Eq. (2.3). In our investigation we took advantage of results of the NNN system, where such states were associated with a particular subset of values of $\kappa$ : those values whose four-adic expression is characterized by an infinite periodic tail. So we concentrated our attention on several states where $\kappa$ belongs to the subset referred to, especially on those values where the exotic character was more evident (e.g., $\kappa=4 / 10,7 / 10,4 / 7$ ). Our numerical investigation points toward the existence of critical states: the evaluation of the band/gap ratio (up to the generation $N=25$ ) converges to a finite value, showing that the band splitting leads locally to a self-similar subband structure, which characterizes the presence of critical states. This prediction is confirmed by the integration of the Eq. (2.3), which shows the presence of several bursts of increasing amplitude, separated by very large distances, as illustrated in Fig. 3.

\section{CONCLUSIONS}

In this paper we have presented an investigation of the properties of spectrum and wave functions of a tight-binding Hamiltonian on the Koch curve with on-diagonal factors in specific sites. Exact analytical results have been obtained within the scheme of periodic approximations and the use of the TM formulation of the pertinent Schrödinger equation. The results have been also confirmed by the numerical integration of this equation.

This work follows a series of previous investigations of a NNN model on the Koch fractal. In the former case we employed not only the methods quoted above, ${ }^{25,26}$ but also a renormalization procedure for the exact evaluation of Green's function and the density of states. ${ }^{27}$ The present model has many interesting features of its own, both in re- spect to the spectrum and to wave functions. However, since results were available for the NNN model, our main interest was to obtain a deeper insight into the properties of quantum Hamiltonians on nonramified fractal substrates, identifying the features of the states which are directly linked to the geometry of the fractal and those which depend directly on the decoration of the model.

We found that the band splitting process depends actually only on the geometry of the fractal since it proceeds according to the same scheme which was identified in the case of the NNN system. We have also shown that a classification of the nature of the states in dependence on the value of $\kappa$ can also be made with the help of the four-adic expression of the value of $\kappa$. This suggests that the classification of wave functions and the band splitting process are generic for any tightbinding model on the Koch fractal. Also, the argumentation can be easily extended to the analysis of similar models on any nonbranching fractal.

On the other hand, the particular classification scheme of the quantum states is certainly model dependent. This is clearly evidenced by a comparison of the invariant sets of the maps (2.8) and their correspondent for the NNN system: the FP's here are the same as those of the linear chain, and indicate the presence of extended states while they are linked to exotic states in the NNN system.

For the purpose of comparison of the classification of states in the two models, let us take first the states on the band edges, which have a finite tail in the four-adic expression of $\kappa$. In the NNN system almost all such states were exponentially localized. Now part of these states is extended and this character is explained by the fact that their corresponding orbit is attracted by one FP. Another subset is formed by such states having localized character. Finally, we have identified a third set of states on band edges, whose main feature is that they diverge faster than any power of $N$. This set is also related to a subset of extended states, and, to our knowledge, this kind of behavior is completely new in the context of quantum states of quasiperiodic onedimensional operators.

The evaluation of the band-gap ratio indicates the exis- 
tence of critical states for several values of $\kappa$ which also admit similar states in the NNN system. The integration of the Schrödinger equation also reveals the existence of critical states, although this fact cannot be directly derived from the renormalization equations as in the former model.
To conclude we state that the investigation of this model indicates the presence of extended, localized, and critical states, all of which seem to be dense along the $\kappa$ axis. This behavior is different from that of the NNN system, where only localized and critical states were found to be dense.
${ }^{1}$ B.B. Mandelbrot, The Fractal Geometry of Nature (Freeman, New York, 1983).

${ }^{2}$ K. Falconer, Fractal Geometry (Wiley, Chichester, 1990).

${ }^{3}$ G. Andre and S. Aubry, Ann. Isr. Phys. Soc. 3, 133 (1980).

${ }^{4}$ J.B. Sokoloff, Phys. Rep. 126, 189 (1985).

${ }^{5}$ M. Kohmoto, L.P. Kadanoff, and C. Tang, Phys. Rev. Lett. 50, 1870 (1983); S. Ostlund, R. Pandit, D. Rand, H.S. Schellnhuber, and E.D. Siggia, ibid., 50, 1873 (1983).

${ }^{6}$ J. Bellissard, D. Bessis, and P. Moussa, Phys. Rev. Lett. 49, 701 (1982)

${ }^{7}$ Y. Gefen, B.B. Mandelbrot, and A. Aharony, Phys. Rev. Lett. 45, 855 (1980).

${ }^{8}$ D.R. Grempel, S. Fishman, and R.E. Prange, Phys. Rev. Lett. 49, 833 (1982).

${ }^{9}$ T. Odagaki and H. Aoyama, Phys. Rev. Lett. 61, 775 (1988).

${ }^{10}$ M. Kohmoto, Phys. Rev. B 34, 5043 (1986).

${ }^{11}$ J.P. Lu, T. Odagaki, and J.L. Birman, Phys. Rev. B 33, 4809 (1986).

${ }^{12}$ J. Moser, Commentat. Math. Helv. 56, 198 (1981).

${ }^{13}$ M. Kohmoto, B. Sutherland, and C. Tang, Phys. Rev. B 35, 1020 (1987).

${ }^{14}$ B. Sutherland and M. Kohmoto, Phys. Rev. B 36, 5877 (1987).

${ }^{15}$ M. Kohmoto and Y. Oono, Phys. Lett. 102A, 145 (1984).

${ }^{16}$ T. Fujiwara, M. Kohmoto, and T. Tokihiro, Phys. Rev. B 40, 7413 (1989).
${ }^{17}$ C.E.T. Gonçalves da Silva and B. Koiller, Solid State Commun. 40, 215 (1981).

${ }^{18}$ S. Alexander, Phys. Rev. B 29, 5504 (1984).

${ }^{19}$ R. Rammal, J. Phys. (Paris) 45, 191 (1984).

${ }^{20}$ A.-M. S. Tremblay and B.W. Southern, J. Phys. (Paris) Lett. 44, L843 (1983).

${ }^{21}$ J.-M. Langlois, A.-M. S. Tremblay, and B.W. Southern, Phys. Rev. B 28, 218 (1983).

${ }^{22}$ W.A. Schwalm, C.C. Reese, C.J. Wagner, and M. K. Schwalm, Phys. Rev. B 49, 15650 (1994).

${ }^{23}$ J. Ashraff and R.B. Stinchcombe, Phys. Rev. B 37, 5723 (1988).

${ }^{24}$ R.B. Capaz, B. Koiller, and S.L.A. de Queiroz, Phys. Rev. B 42, 6402 (1990).

${ }^{25}$ R.F.S. Andrade and H.S. Schellnhuber, Europhys. Lett. 10, 73 (1989).

${ }^{26}$ P. Kappertz, R.F.S. Andrade, and H.J. Schellnhuber, Phys. Rev. B 49, 14711 (1994).

${ }^{27}$ R.F.S. Andrade and H.J. Schellnhuber, Phys. Rev. B 44, 13213 (1991).

${ }^{28}$ M. Kohmoto and J. R. Banavar, Phys. Rev. B 34, 563 (1986).

${ }^{29}$ R.F.S. Andrade and S.R. Salinas, J. Phys. A 17, 1665 (1984).

${ }^{30}$ R. Johnson and J. Moser, Commun. Math. Phys. 84, 403 (1982).

${ }^{31}$ B. Simon, Adv. Appl. Math. 3, 463 (1982).

${ }^{32}$ H.J. Schellnhuber and H. Urbschat, Phys. Rev. Lett. 54, 588 (1985). 